\title{
Magnetization transfer and diffusion imaging of acute axonal damage in the cerebral peduncle following hypoxia-ischemia in neonatal rats
}

\author{
Ursula I. Tuor'-3, Min Qiao', Melissa Morgunov' ${ }^{1}$, Emily Fullerton' ${ }^{1}$, Tadeusz Foniok² and Adam Kirton ${ }^{2-4}$
}

BACKGROUND: Magnetic resonance imaging (MRI) of axonal degenerative changes in the cerebral peduncle of the corticospinal tract following cerebral hypoxic-ischemic damage might distinguish infants most appropriate for receiving prompt treatment. The optimal MRI sequence for very early diagnosis of axonal degenerative changes is unknown. We hypothesized that magnetization transfer ratio (MTR) imaging would be more sensitive than traditional MRI, e.g., T 2 or diffusion weighted imaging. METHODS: Transient unilateral cerebral hypoxia-ischemia was produced in the neonatal rat followed by MRI of changes in $T_{2}$, the apparent diffusion coefficient (ADC) of water, and $M T R$, with a focus on the parietal cortex (an ischemic damaged region) and the cerebral peduncle (remote within the corticospinal tract). Rats were imaged at $2 \mathrm{~h}, 1 \mathrm{~d}$, or $1 \mathrm{wk}$ postinsult. RESULTS: In the cerebral peduncle, MTR and $T_{2}$ responded similarly, with alterations occurring ipsilaterally at $1 \mathrm{~d}$ postinsult. ADC was most sensitive for detecting changes as early as $2 \mathrm{~h}$ postinsult, and this corresponded to a reduced staining of axonal filaments ipsilaterally.

CONCLUSION: MTR and $T_{2}$ imaging have comparable sensitivity for distinguishing early axonal damage in the cerebral peduncle. ADC imaging is highly sensitive for detecting early disruption of corticospinal axons, supporting its potential hyperacute diagnostic use clinically.

P erinatal hypoxic-ischemic brain injury and perinatal stroke are leading causes of death and neurological disability (1-3). Considering the lifelong suffering and high costs to society, there is an urgent need for both effective new therapies and improved early diagnosis to help select patients who would benefit from confirmed therapies such as hypothermia (4). In addition, an improved understanding of early pathological processes is needed to identify therapeutic targets when treatment opportunities are greatest (5). Magnetic resonance imaging (MRI) (e.g., $\mathrm{T}_{2}$ - or diffusion-weighted imaging) is the main tool for the diagnosis of hypoxic-ischemic encephalopathy or stroke, and its use is possible within the immediate perinatal time frame (3,5-9).
Determination of MRI abnormalities such as lesion size and location shows consistent but relatively weak associations with long-term outcomes $(3,10,11)$. By contrast, function-specific imaging biomarkers may have greater potential to predict outcomes. The leading example is early diffusion imaging changes in the descending corticospinal tract, which appear to correlate well with motor outcome in neonatal (e.g., cerebral palsy), childhood, and adult stroke $(12,13)$. We have shown recently, using MRI in a neonatal model of hypoxia-ischemia, that similar to changes in the descending corticospinal tract in human infants, there are acute (by $24 \mathrm{~h}$ ) increases in $\mathrm{T}_{2}$ and decreases in the apparent diffusion coefficient (ADC) of water, consistent with early Wallerian degeneration (14). Earliest possible detection of these changes is desirable for maximal clinical utility.

An alternative MRI scan, a magnetization transfer (MT) sequence, uses radiofrequency pulses that are "off" or "on" resonance to probe the properties of free and bound water, producing a magnetization transfer ratio (MTR) image (15-17). MR scans with MT contrast have been reported to be more sensitive than $\mathrm{T}_{2}$ imaging for detecting acute mild ischemic changes in white matter (18) or chronic degenerative changes in adult axons (19-22). Whether alternative MR methods such as MT imaging are capable of detecting axonal degeneration in earlier, hyperacute time frames (e.g., $<6 \mathrm{~h}$ ) is unknown.

We hypothesized that MT imaging would be more sensitive than $\mathrm{T}_{2}$ or diffusion imaging for detecting early signs of axonal injury in perinatal hypoxic-ischemic brain injury. We used our neonatal rat model of transient unilateral cerebral hypoxiaischemia to investigate time points as early as $2 \mathrm{~h}$ postinsult and compared the changes in $T_{2}, A D C$, and MTR. We focused on changes occurring in the cerebral cortex (an ischemic region during the hypoxia-ischemia) and the cerebral peduncle (a region within the descending corticospinal tract remote from the site of the ischemia caused by carotid artery ligation and hypoxia). We demonstrate that following a unilateral cerebral hypoxicischemic insult, MTR was comparable with $\mathrm{T}_{2}$ but less sensitive than ADC for detecting early corticospinal tract changes, and 
these hyperacute changes were associated with altered staining of axonal filaments in the cerebral peduncle.

\section{RESULTS}

MRI intensity changes in MT, $\mathrm{T}_{2}$-weighted, and diffusionweighted images were observed ipsilateral to the transient hypoxia-ischemia (Figure 1), whereas no ipsilateral-contralateral differences were observed in sham-treated animals. Parenchymal lesions were evident within a brain distribution consistent with the P7 model of unilateral transient cerebral hypoxia-ischemia $(23,24)$. Cortical, striatal, and thalamic ischemic changes were observed consistently in all animals (lesion volume of $37.1 \pm 7.8 \%$ of the hemisphere at $1 \mathrm{~d}$ after hypoxia-ischemia). At $2 \mathrm{~h}$ postinsult, ischemic damaged areas, such as the cerebral cortex, demonstrated an enhanced ipsilateral signal intensity in both $\mathrm{T}_{2}$ - and diffusion-weighted images (Figure $1 \mathrm{~b}, \mathrm{c}$ ) and a decreased intensity in MT ratio images (Figure 1a). In five of seven animals (71\%) at $2 \mathrm{~h}$ postinsult, increases in intensity in diffusion-weighted images were also detected in posterior subcortical brain remote from the site of the ischemia, specifically within the cerebral peduncle of the descending corticospinal tract (Figure 1f). By $1 \mathrm{~d}$ postinsult, intensity changes were observed for all scan types, both in directly injured ischemic regions such as the cerebral cortex and within the posterior cerebral peduncle of the descending a

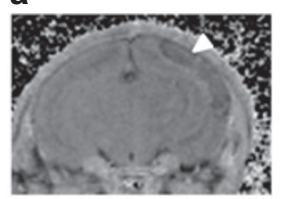

d

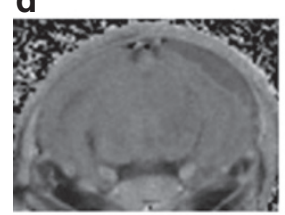

g

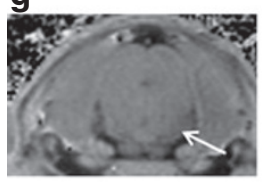

j

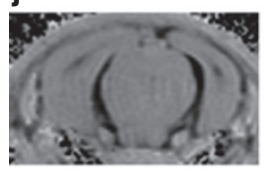

b

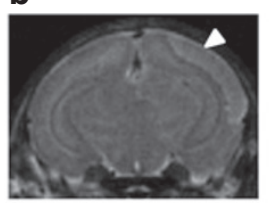

e

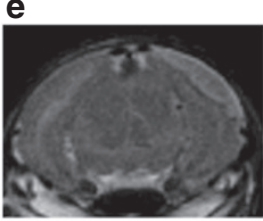

$\mathbf{h}$

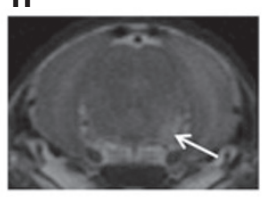

k

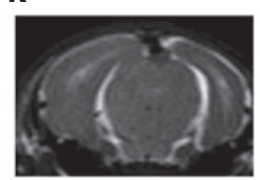

C

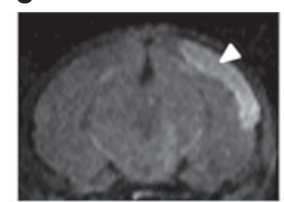

f
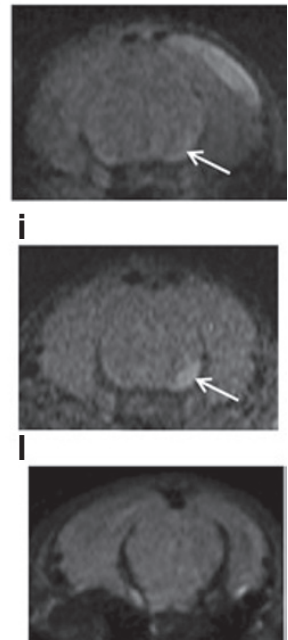

Figure 1. Representative MRI scans of the brain $(\mathbf{a}-\mathbf{c})$ at an anterior level and (d-I) at the level of the pons. Images are from a neonatal rat at different times $((\mathbf{a}-\mathbf{f}) 2 \mathrm{~h},(\mathbf{g}-\mathbf{i}) 1 \mathrm{~d}$, or $(\mathbf{j}-\mathbf{l}) 1 \mathrm{wk})$ after an episode of unilateral (right) cerebral hypoxia-ischemia (HI). Localized magnetic resonance changes are visible at acute times after HI. (a, d, $, \mathbf{g}, \mathbf{j})$ MTR images, $(\mathbf{b}, \mathbf{e}, \mathbf{h}, \mathbf{k})$ $\mathrm{T}_{2}$-weighted images, and $(\mathbf{c}, \mathbf{f}, \mathbf{i}, \mathbf{l})$ diffusion-weighted images. Alterations in image intensity were detected within regions such as the cerebral peduncle (arrows) and parietal cortex (arrowheads). MRI, magnetic resonance imaging; MTR, magnetization transfer ratio. corticospinal tract (Figure 1g-i). At 1 wk postinsult, intensity levels in MR scans were similar, and differences between left and right hemispheres were difficult to distinguish (Figure $\mathbf{1} \mathbf{j}-\mathbf{l}$ ).

Quantification of the MR scans confirmed significant ipsilateral-contralateral differences at acute and subacute times after hypoxia-ischemia (Figure 2). Diffusion-weighted changes, quantified by determining changes in the ADC for water, were significant at $2 \mathrm{~h}$ after hypoxia-ischemia in the parietal cortex and cerebral peduncle. At $1 \mathrm{~d}$ after hypoxia-ischemia, there were ipsilateral changes in MTR (Figure 2a), $\mathrm{T}_{2}$ (Figure 2b), and ADC (Figure 2c) within the parietal cortex and the cerebral peduncle. These normalized by $1 \mathrm{wk}$ after hypoxia-ischemia. Within the pons, MTR, $\mathrm{T}_{2}$, and ADC were similar in the ipsilateral and contralateral regions at all time points investigated.

Sensitivity of the MTR sequence for detecting changes was assessed by comparing the mean ipsilateral vs. contralateral differences within the different regions of interest (Figure 3). All sequences were sensitive for detecting alterations in ischemic lesions such as the cerebral cortex at $2 \mathrm{~h}$ and $1 \mathrm{~d}$ postinsult. In cortical tissue, the mean changes in MTR tended to be less marked than those in $\mathrm{T}_{2}$. Within the cerebral peduncle acutely $(2 \mathrm{~h}$ after hypoxia-ischemia), ADC was the most sensitive in detecting the alterations as compared with MTR and $\mathrm{T}_{2}$. At $1 \mathrm{~d}$ after hypoxiaischemia, all sequences detected changes in cerebral peduncle ipsilaterally, with ADC having a significantly greater relative change than MTR. At $1 \mathrm{wk}$ postinsult, the ipsilateral vs. contralateral differences in $\mathrm{T}_{2}, \mathrm{MTR}$, and ADC normalized.

The observed changes in the cerebral peduncle were not associated with the severity of the ischemic insult. At any time after hypoxia-ischemia, there was a lack of a correlation (Pearson product moment) between the ischemic lesion volumes and the changes in MTR, $\mathrm{T}_{2}$, or ADC. There was also no correlation between the changes in the MR values measured within the cerebral peduncle as compared with those in the parietal cortex, a region directly injured by the hypoxia-ischemia.

Histological changes in regions corresponding to those assessed with MRI were investigated. Standard hematoxylin and eosin staining demonstrated areas of infarct (e.g., within the parietal cortex) and more subtle edematous changes associated with vacuolation or paler eosin staining in regions such as the cerebral peduncle (Figure $4 a-c$ ). Measurement of optical gray levels in the regions of interest demonstrated a significantly lighter staining ipsilaterally as compared with that contralaterally at $2 \mathrm{~h}$ and $1 \mathrm{~d}$ after hypoxia-ischemia within the cerebral peduncle and at all times within the cortex (Figure 4d). In SMI31-immunostained sections, positive staining was less intense and rather diffuse in immature rats (7-d-old rat brain) as compared with that in 2-wk-old rats. Heterogeneous positive staining intensity was similar in the left and right hemispheres in sham-treated controls. There was a reduced SMI31 staining within the subcortical posterior cerebrum ispilateral to the hypoxia-ischemia (Figure 4e,f). Ipsilateral-contralateral SMI31 staining was significantly different at all times after hypoxia-ischemia in the axons of the cerebral peduncle but not in the gray matter regions of the pons or parietal cortex (Figure 4d). 
a
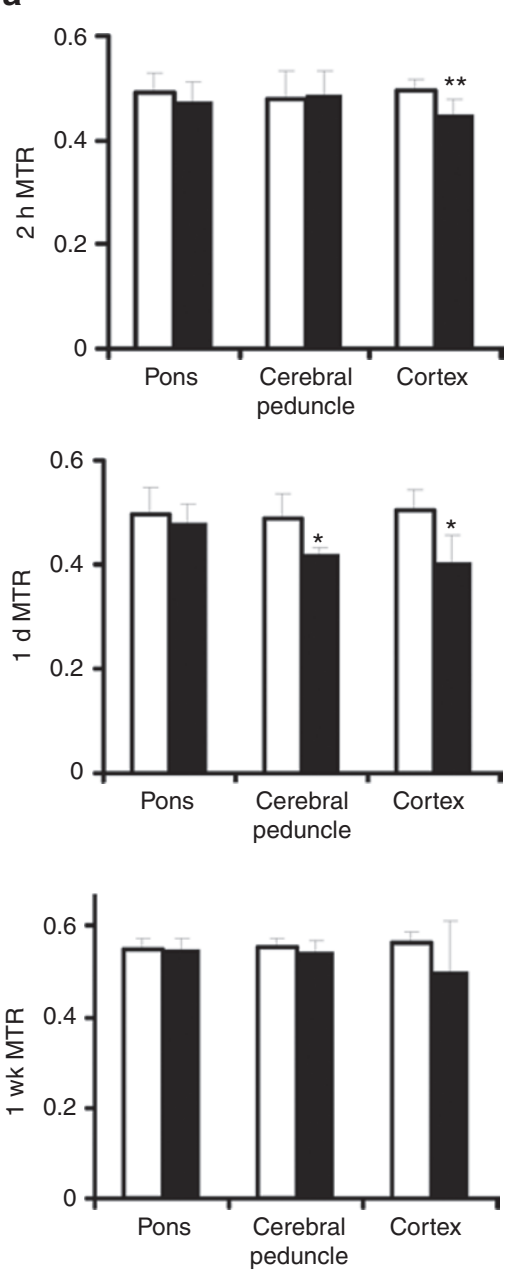

b
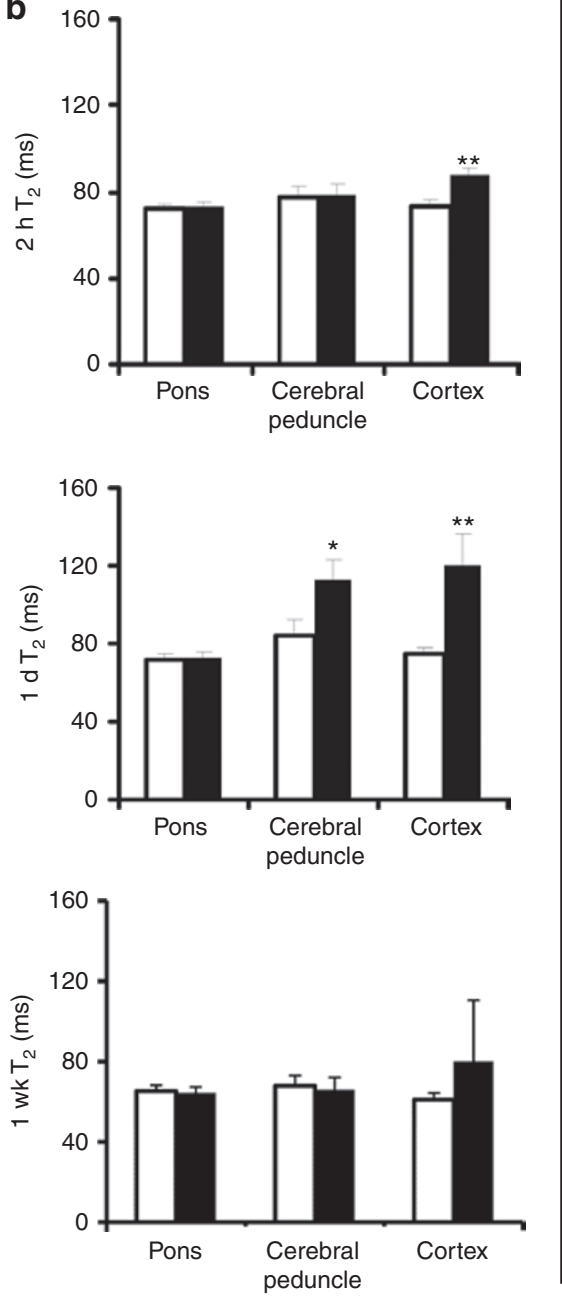

C
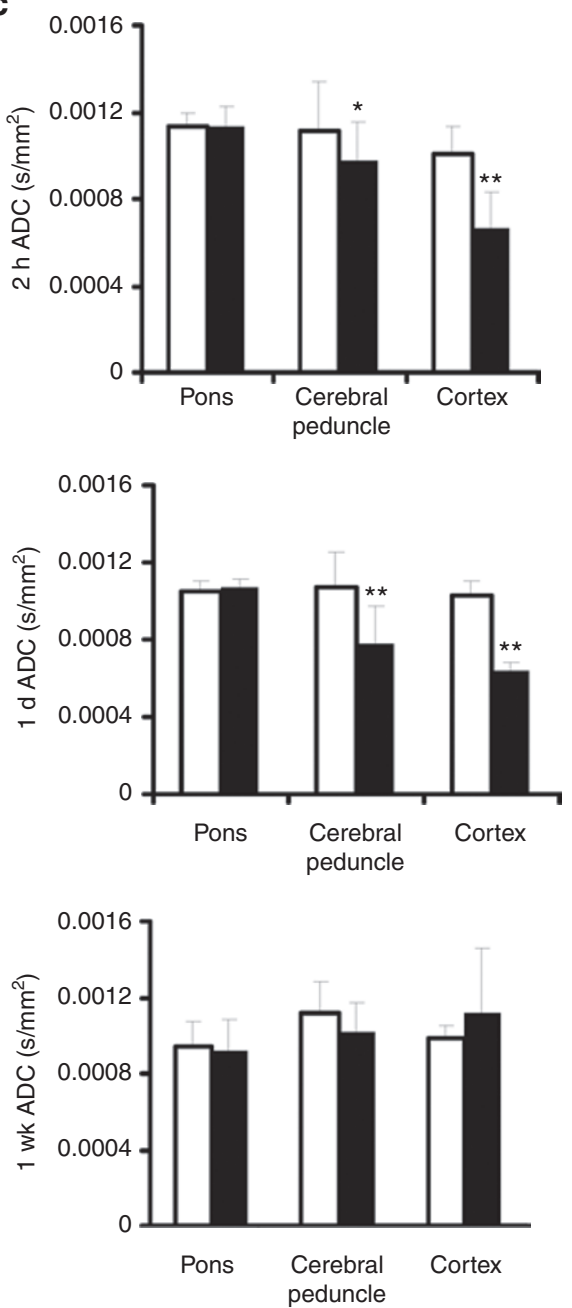

Figure 2. Quantitative imaging changes in the pons, parietal cortex, and cerebral peduncle (within the corticospinal tract) measured ipsilateral (white bars) and contralateral (black bars) to the insult. Mean values for (a) the MTR, (b) $\mathrm{T}_{2}$, and (c) ADC are shown for the regions of interest at $2 \mathrm{~h}$ (top row), $1 \mathrm{~d}$ (middle row), or $1 \mathrm{wk}$ (bottom row) following an episode of unilateral cerebral hypoxia-ischemia. Significant mean ipsilateral as compared with contralateral differences $\left({ }^{*} P<0.05,{ }^{* *} P<0.005\right)$ are observed within the parietal cortex and cerebral peduncle. ADC, apparent diffusion coefficient; MTR, magnetization transfer ratio.
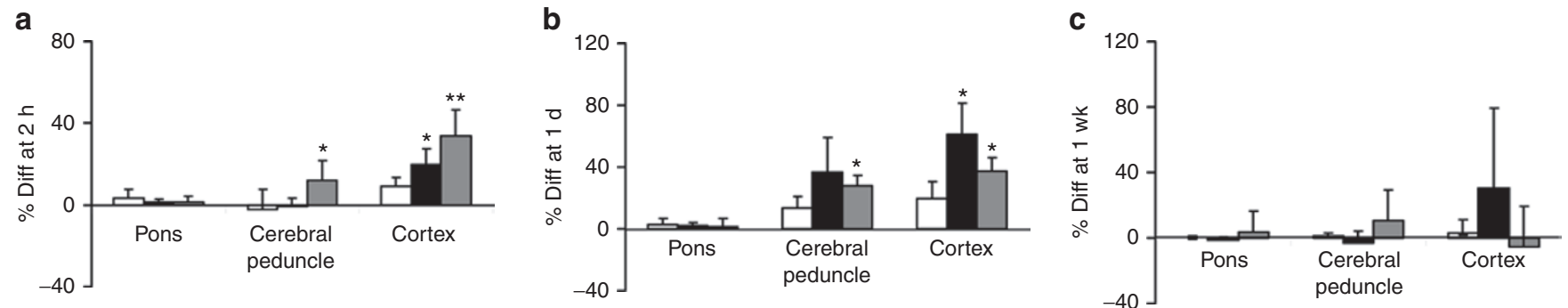

Figure 3. Comparison of ipsilateral vs. contralateral difference (Diff) for measures of the MTR (white bars), $T_{2}$ (black bars), and ADC (gray bars). Shown are the mean values $( \pm S D)$ for the hemispheric differences in magnitude for $T_{2}$ (ipsilateral - contralateral/contralateral percentage) or MTR and ADC (contralateral - ipsilateral/contralateral percentage) at (a) $2 \mathrm{~h}$, (b) $1 \mathrm{~d}$, and (c) $1 \mathrm{wk}$ following an episode of unilateral cerebral hypoxia-ischemia. The regions examined include the pons, cerebral peduncle, and parietal cortex. In the cerebral peduncle, ipsilateral vs. contralateral differences in mean $\mathrm{T}_{2}$ and $\mathrm{MTR}$ were similar, whereas ipsilateral vs. contralateral differences in ADC differed from those in MTR $(* P<0.05, * * P<0.005$, different from MTR, ANOVA and a Bonferroni multiple comparison). ADC, apparent diffusion coefficient; MTR, magnetization transfer ratio.

\section{DISCUSSION}

This study in neonatal rats is the first to our knowledge to examine the time course and sensitivity of MTR imaging for detecting early corticospinal tract degeneration following cerebral ischemic injury. The results have identified several original findings. First, we observed a decrease in MTR at an acute time of $1 \mathrm{~d}$ following a hypoxic-ischemic insult in the descending corticospinal tract remote from the direct ischemic injury, such as 
a

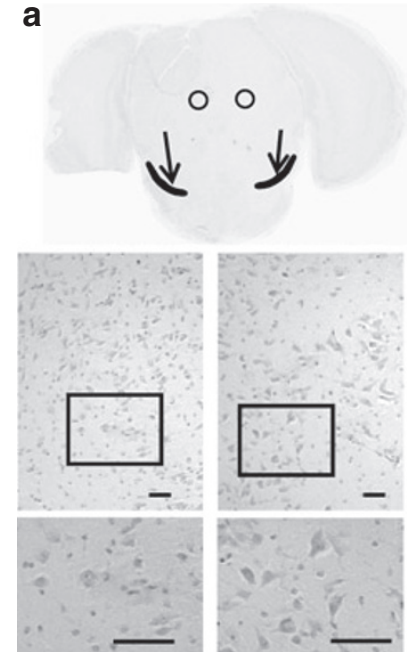

b
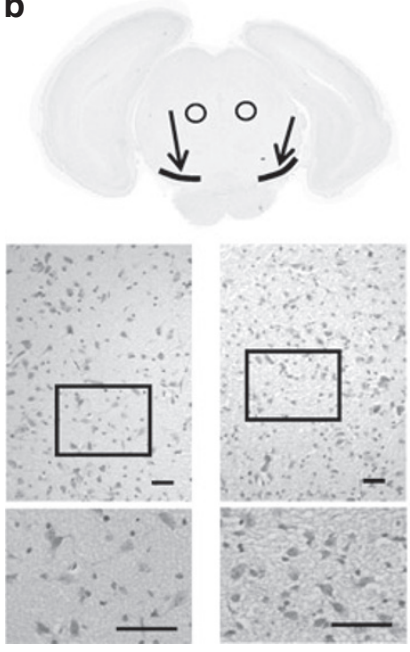

C
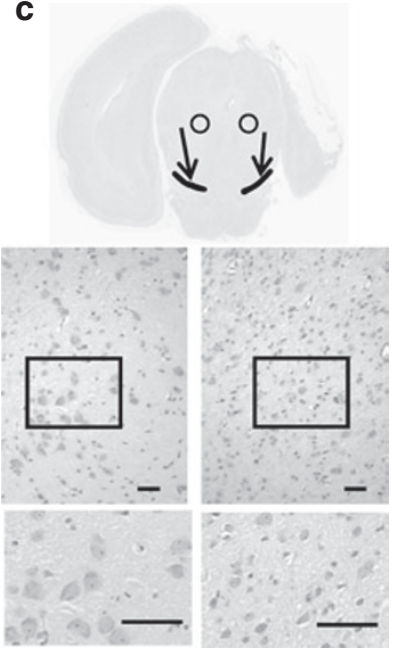

$\mathbf{f}$

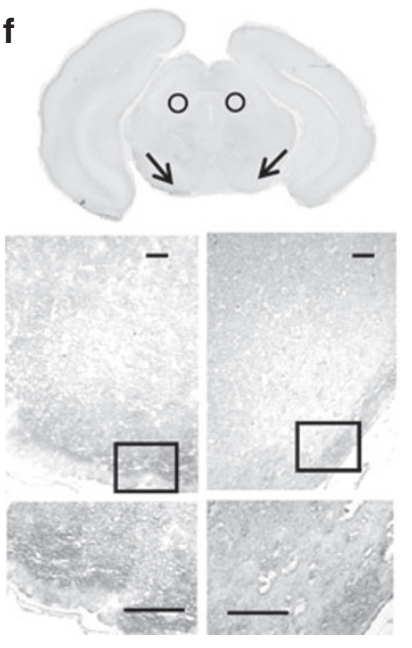

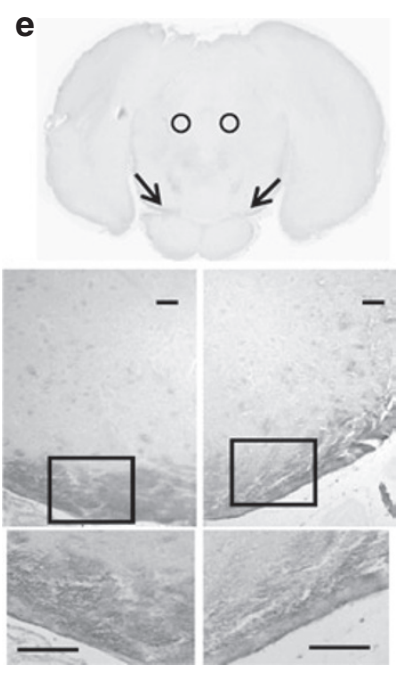

g

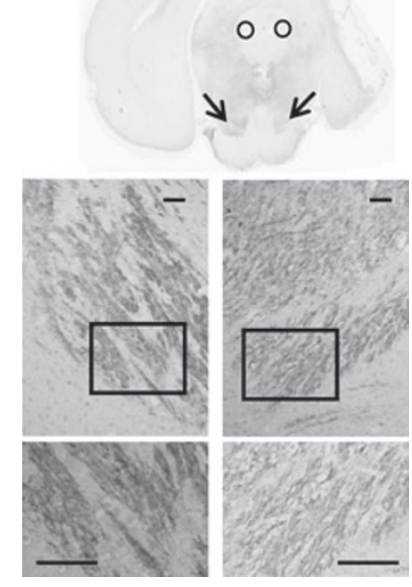

Figure 4. (a-c) Representative sections stained with hematoxylin and eosin (HE) or (e-f) sections stained immunohistochemically for axonal filaments using SMI31, from animals killed at $(\mathbf{a}, \mathbf{e}) 2 \mathrm{~h},(\mathbf{b}, \mathbf{f}) 1 \mathrm{~d}$, or $(\mathbf{c}, \mathbf{g}) 1 \mathrm{wk}$ after cerebral hypoxia-ischemia. (d) Mean optical intensity measures from the HEand SMI31-stained sections are shown for regions of interest that include the pons (e.g., circles) and cerebral peduncle (e.g., filled curves and arrows). Mean values $( \pm$ SD) for different times at $2 \mathrm{~h}$ (white bars), $1 \mathrm{~d}$ (black bars), and $1 \mathrm{wk}$ (gray bars) after insult are presented as a difference in ipsilateral and contralateral hemispheres converted to a percentage of the contralateral value. Shown below each of the representative micrographs are magnified images taken for the left and right cerebral peduncle at the arrows and then further magnified within the rectangles. Bar is $100 \mu \mathrm{m}$. ${ }^{*} P<0.05,{ }^{* *} P<0.01$, ipsilateral value different from contralateral value, using a paired Student's $t$-test.

that in the cerebral cortex. Second, the results demonstrated that MT imaging is no more sensitive than $\mathrm{T}_{2}$ imaging for diagnosing the distal axonal changes in the corticospinal tract following cortical ischemic damage. Third, we found that diffusion imaging and ADC appear to be highly sensitive for detecting hyperacute changes within the ipsilateral cerebral peduncle at $2 \mathrm{~h}$ after hypoxic-ischemic injury. Finally, the ADC decreases within the cerebral peduncle at early times postinsult corresponded to paler hematoxylin and eosin staining ipsilaterally and reduced positive staining for axonal neurofilaments with SMI31. MTR provided no additional advantage over more standard imaging methods, and ADC was the most sensitive method for the detection of very early remote axonal injury following cerebral ischemia produced by right carotid artery ligation and hypoxia.

The model of transient unilateral cerebral hypoxia-ischemia used in the current study produces a substantial unilateral infarction with a well-characterized distribution of damage. Ischemic damage is known to occur ipsilateral to the carotid artery ligation in the regions of the cerebral cortex, hippocampus, thalamus, and striatum within the middle cerebral artery territory. MRI changes in this model are also well characterized within the regions that develop infarcts, such as the cerebral cortex. White matter structures such as the internal capsule may also receive ischemic damage, but, of importance, posterior brainstem and pontine structures including the cerebral peduncle are not ischemic in this model (23). In ischemic damaged regions at $1 \mathrm{~d}$ postinsult, there are increases in $\mathrm{T}_{2}$ associated with vasogenic edema and decreases in ADC that have been shown to be associated with a cell swelling or cytotoxic edema $(25,26)$. Therefore, acutely there is a combination of vasogenic and cytotoxic edema in ischemic injured brain areas such as the parietal cortex. Normalization of such $\mathrm{T}_{2}$ and ADC changes by $1 \mathrm{wk}$ 
postinsult has been reported previously and was confirmed in the current study (14). Few studies have investigated the 2-h time point, although similar cortical increases in $\mathrm{T}_{2}$ and decreases in ADC have been observed at $1 \mathrm{~h}$ (27). It should be noted that there can be some variability in the regional distribution, magnitude, and progression of changes, depending on the severity of the hypoxia-ischemia, with mild and substantial insults having different profiles (18). In the current study, the duration of unilateral cerebral hypoxia-ischemia was moderate, resulting in a fairly consistent volume of ischemic damage. Moreover, it is important to acknowledge that measures of MTR are affected by edematous changes within ischemic lesions such that at more chronic time points (e.g., $1 \mathrm{wk}$ or longer), MTR can be markedly reduced within slices containing cysts. Cystic regions were avoided by measuring pericystic or peri-infarct regions, and in these peri-infarct regions, MTR values were normalized by $1 \mathrm{wk}$ after hypoxia-ischemia.

Progression of standard $\mathrm{T}_{2}$ and $\mathrm{ADC}$ sequence imaging changes within the descending corticospinal tract has been reported recently using the same animal model (14). In this study, we investigated the imaging changes between $1 \mathrm{~d}$ and $4 \mathrm{wk}$ postinsult, and similar to the present results, decreases in ADC and increases in $\mathrm{T}_{2}$ were observed within the cerebral peduncle at $1 \mathrm{~d}$ postinsult, with a return toward sham-treated control or contralateral values by $1 \mathrm{wk}$ (14). These MR changes were associated with early signs of Wallerian degeneration as detected by altered staining of axons using a silver stain and SMI31 immunohistochemistry. The current study expands these findings by investigating the standard imaging changes in the cerebral peduncle at a hyperacute time point $(2 \mathrm{~h})$ after injury. We found that $\mathrm{T}_{2}$ in the ipsilateral cerebral peduncle was similar to that in the contralateral cerebral peduncle at this very early imaging time point. However, hyperintense changes were already visible in the cerebral peduncle in diffusion-weighted images, corresponding to a significant ipsilateral decrease in ADC suggestive of cytotoxic edema. In histological sections stained for axonal neurofilaments or with hematoxylin and eosin, there was a decrease in staining ipsilaterally at this early and subsequent time points. This is consistent with early degenerative changes in axons that have been observed as soon as $30 \mathrm{~min}$ after transection of axons in mouse spinal cord in vivo (28). Within $300 \mu \mathrm{m}$ from the site of transection, there is initially beading and swelling of the axon membrane. This is followed by continued narrowing of the axon into fragments and its subsequent disintegration or dieback within hours. At around $30 \mathrm{~h}$, this is followed by further fragmentation and subsequent disintegration of the distal end of the axon. A similar sequence of axonal swelling focally, that subsequently progresses to axonal fragmentation, also occurs in a mouse model of experimental autoimmune encephalomyelitis with focal axonal degeneration (29). The current study supports the notion that a similar axonal degeneration is produced following hypoxic-ischemic damage to the cerebral cortex. In the cerebral peduncle, the early axonal swelling and fragmentation reported by others $(28,29)$ appear to be detected at $2 \mathrm{~h}$ as a reduction in ADC and paler hematoxylin and eosin and SMI31 staining, which by $1 \mathrm{~d}$ is also mixed with a vasogenic edema reflected by increased $\mathrm{T}_{2}$.
We had hypothesized that MT imaging would be particularly sensitive for detecting changes, within white matter tracts, associated with axonal degeneration following transient unilateral cerebral hypoxia-ischemia in the neonate. MT imaging was able to detect a significant reduction in MTR in the posterior cerebral peduncle of the descending corticospinal tract, but this was seen only by the day 1 time point, similar to the observed changes in $\mathrm{T}_{2}$. Clinically, MT imaging has detected changes in the corticospinal tract of patients with a number of different neurodegenerative diseases, and in contrast to the findings of the current study, several others have reported a greater sensitivity or reliability of MT than $\mathrm{T}_{2}$ contrast imaging. For example, in patients with amyotrophic lateral sclerosis, a decreased MT ratio was observed in the pyramidal tract, yet the majority of subjects had normal $T_{2}$ imaging $(19,20)$. Similarly, in studies of patients with amyotrophic lateral sclerosis or probable multiple system atrophy, imaging with a $\mathrm{T}_{1}$ spin echo sequence with MT contrast consistently showed altered intensity within the pyramidal tract, despite normal-appearing $\mathrm{T}_{2}$ contrast in fluid-attenuated inversion recovery images $(21,30)$. One major difference between these clinical studies and the current study is the timing of the degenerative process being imaged, which is likely more chronic (e.g., weeks rather than days) in the clinical investigations. It is also possible that the axonal changes following stroke or hypoxia-ischemia differ from those in slowly progressing neurodegenerative diseases or that there are differences in immature as compared with adult brain. Irrespective of the reasons for different results, the current findings indicate that both MT and $\mathrm{T}_{2}$ imaging have similar sensitivity for detecting axonal changes within the cortiocospinal tract occurring in the first week following cerebral hypoxia-ischemia in the neonate.

In conclusion, a need for early accurate diagnosis of brain injury following perinatal stroke/hypoxia-ischemia is gaining importance in facilitating appropriate and rapid decisions regarding infant management and treatment. Selecting patients for treatment studies might be guided most effectively by using signs of ischemic injury that are indicative of poor outcome such as Wallerian degeneration $(12,13)$. Our comparison of $\mathrm{MT}, \mathrm{T}_{2}$, and ADC imaging of the corticospinal tracts following cerebral hypoxic-ischemic damage in the neonate demonstrates that MTR detects subacute degenerative changes, but these reductions in MTR ratio are similar to increases in $\mathrm{T}_{2}$. Indeed, there is no added diagnostic benefit of MT ratio imaging for identifying corticospinal tract abnormalities $2 \mathrm{~h}$ to $1 \mathrm{wk}$ after a hypoxic-ischemic infarct. $\mathrm{ADC}$ is the most sensitive MR sequence for distinguishing axonal injury in the corticospinal tract, with hyperacute imaging changes observed as early as $2 \mathrm{~h}$ postinsult. Hyperacute ADC imaging of early Wallerian degeneration holds promise for further study as a method of selecting infants most likely to benefit from new or current treatments, such as hypothermia.

\section{METHODS}

\section{Animal Model of Hypoxia-Ischemia}

The procedures followed in the use of animals were in accordance with the guidelines of the Canadian Council on Animal Care and were approved by the University of Calgary Animal Care Committee. Twenty-one male or female rat pups from eight litters delivered from 
Wistar females (Charles River Laboratories, Montreal, Canada) were randomized to cerebral hypoxia-ischemia groups ( $n=6-7$ per group) destined for MRI at $2 \mathrm{~h}, 1 \mathrm{~d}$, or $1 \mathrm{wk}$ after surgery as described previously $(14,18)$. Briefly, on the seventh day of life, animals in the transient unilateral cerebral hypoxia-ischemia group $(n=21)$ were anesthetized with $2 \%$ isoflurane in $30 \%$ oxygen, and the right common carotid artery was exposed surgically, ligated, and transected. This was followed by infiltration of the wound with a local analgesic ( $0.25 \%$ bupivacaine; Ayerst Veterinary Laboratory, Guelph, Ontario, Canada) and closure of the incision with tissue adhesive (Permabond 910; Electron Microscopy Sciences, Hatfield, PA) and a suture. Following 30-min recovery from anesthesia, animals were returned to the dam for $1-1.5 \mathrm{~h}$ before exposure to $65 \mathrm{~min}$ of hypoxia $\left(8 \% \mathrm{O}_{2}, 92 \% \mathrm{~N}_{2}\right)$ in a chamber controlled at $35.5^{\circ} \mathrm{C}$. There was an adequate collateral blood supply ipsilateral to the ligation except during exposure to hypoxia. During hypoxia, the reduction in blood flow to the ipsilateral hemisphere within the distribution of the middle cerebral artery territory becomes sufficiently ischemic to produce permanent damage and, essentially, a transient unilateral cerebral hypoxia-ischemia $(23,24)$. Sham-treated control pups $(n=15)$ underwent a similar procedure without carotid artery ligation or exposure to hypoxia.

\section{MRI}

At the time of MRI, animals were anesthetized with isoflurane (1.5-2.5\%) and scanned within a 9.4T MRI system (Biospin, Bruker, Germany) using methods similar to those described previously $(14,18)$. Briefly, the head of the rat was positioned within a quadrature volume coil of $3.5 \mathrm{~cm}$ diameter and restrained using a custom-designed headband. Respiration was monitored and maintained normal via adjustments in anesthesia. Animals were maintained normothermic $\left(\sim 37^{\circ} \mathrm{C}\right)$ using a small animal monitoring and servo-controlled feedback heating air system (SA Instruments, Stony Brook, NY).

First acquired were $\mathrm{T}_{2}$ and diffusion scans consisting of $300.5-\mathrm{mm}-$ thick slices, through the medulla and cerebrum. They were acquired using a data matrix size of $128 \times 128$ and field of view of $2.0 \times 2.0 \mathrm{~cm}$. The $\mathrm{T}_{2}$ sequence used to obtain $\mathrm{T}_{2}$ maps consisted of a set of $32 \mathrm{~T}_{2}$-weighted spin echo images with an echo time spacing of $10 \mathrm{~ms}$ between images and a repetition time of $10 \mathrm{~s}$. The sequence to determine ADC maps consisted of a set of diffusion-weighted echo-planar images using five different $b$ values $(74,292,655,891$, and 1,164$)$ acquired with a repetition time of $5,000 \mathrm{~ms}$ and an echo time of $40 \mathrm{~ms}$.

Subsequently, MT imaging scans were acquired to obtain MTR maps. For the MTR map, two-proton density-weighted spin echo scans were acquired consisting of six 0.5 -mm-thick slices with an interslice gap of $0.5 \mathrm{~mm}$ and a matrix size of $128 \times 128$. Scans were obtained with MT saturation either on or off using a repetition time of $6 \mathrm{~s}$, echo time of $15 \mathrm{~ms}$, and 60 saturation pulses with a frequency offset of $1,500 \mathrm{~Hz}$ and a constant radiofrequency power B1 of $6 \mu \mathrm{T}$.

\section{Histology}

After the MR scan, animals ( $n=4$ per group) were deeply anesthetized with pentobarbital $(120 \mathrm{mg} / \mathrm{kg}$, i.p.) and perfused with saline and $10 \%$ formalin. Brains were cut into blocks and embedded in paraffin. Sections $(7 \mu \mathrm{m}$ thick) were cut at the level of the posterior cerebral peduncle in the mid-brain and at the level of the posterior thalamus. The sections were then stained with hematoxylin and eosin in order to assess tissue changes including signs of cell swelling or edema detected by evidence of vacuolation and paler eosin staining. Changes in axonal filaments associated with disintegration of the cytoskeleton were assessed immunohistochemically using SMI31 (Covance, Berkeley, $\mathrm{CA}$ ), an antibody considered to recognize phosphorylated, high-molecular-weight neurofilaments within the axonal cytoskeleton $(31,32)$. Briefly, slides were incubated for $2 \mathrm{~h}$ at room temperature with the primary mouse antibody, SMI31 (1:500), followed by overnight incubation at $4^{\circ} \mathrm{C}$ with biotinylated goat antimouse immunoglobulin $\mathrm{G}(1: 400$; Jackson ImmunoResearch Lab, West Grove, PA). Immune complexes were visualized using 3,3-diaminobenzidine (Sigma, Oakville, Ontario, Canada). Controls included staining of mature rat brain sections containing axons and gray matter and sections processed with omission of the antibody to identify nonspecific staining.

\section{Data Analysis}

Values of T2, MTR, and ADC were measured using locally developed software (Marevisi; National Research Council of Canada, Winnipeg, Manitoba, Canada) within the anatomically specified regions of interest identified using a rat atlas (33) as described previously (14). Regions outlined were areas of the pons (20-25 voxels) and the cerebral peduncle (4-8 voxels) of the descending corticospinal tract at a level of the posterior cerebrum, where the pons was not infarcted (i.e., directly ischemic) following the hypoxia-ischemia. Measures were also taken in the parietal cortex (20-25 voxels) within a slice of the mid-cerebrum, a region consistently damaged in this animal model (14). Regional measures were taken in the hemisphere ipsilateral to the carotid artery ligation, where permanent ischemic damage is generally confined, and the corresponding contralateral homotopic region, where transient hypoxic biochemical changes might occur (24). At $1 \mathrm{wk}$ after hypoxia-ischemia, cystic areas were avoided, and measures were taken within a $0.2-\mathrm{mm}$-wide rim of the adjacent periinfarct parietal cortex. $\mathrm{T}_{2}$ values were obtained by a monoexponential fit of the echo time-related decrease in regional signal intensities in the $\mathrm{T}_{2}$-weighted images. The MTR map was calculated as MTR = $\left(\left(M_{\mathrm{o}}-M_{\mathrm{s}}\right) / M_{\mathrm{o}}\right) \times 100 \%$, where $M_{\mathrm{s}}$ and $M_{\mathrm{o}}$ were the regional signal intensities obtained with and without MT saturation, respectively. ADC was calculated using an exponential fit of the regional intensities in the diffusion-weighted images and their $b$ values. $T_{2}$-weighted images were used to calculate ischemic lesion volume by summing for all slices-the area of hyperintensity $\times$ slice thickness (converted to a percentage of the area of the nonischemic hemisphere).

The histological sections were analyzed for altered levels of SMI31 or hematoxylin and eosin staining. Images of stained sections at the level of the pons, which contained the posterior cerebral peduncle, and at the level of the posterior thalamus, which contained the parietal cortex, were captured digitally using a slide scanner (Coolscan V, version 4; Nikon, Melville, NY). Image J software (National Institutes of Health, Bethesda, MD) was used to convert the images to gray scale. The staining intensity was quantified by measuring the mean optical gray levels within the anatomical areas outlined as for the MR images. Regions of interest were the posterior cerebral peduncle, pons, and parietal cortex in the hemisphere ipsilateral and contralateral to the ischemia produced by right carotid artery ligation and hypoxia. The difference between the contralateral and ipsilateral gray levels was normalized as a percentage of the contralateral value.

Data are reported as mean \pm SD for the different groups studied. Differences between the ipsilateral and contralateral values were compared using a two-tailed Student's paired $t$-test. Differences between groups were compared using an ANOVA and a Bonferroni-corrected $t$-test for multiple comparisons. Statistical differences between means were considered significant at $P<0.05$.

\section{ACKNOWLEDGMENTS}

The authors thank C. Smith and I. Jalal for contributing to preliminary image analysis and histology; $M$. Sule for helping with editing of the figures; and D. Kirk for providing animal anesthesia and monitoring during acquisition of magnetic resonance images.

\section{STATEMENT OF FINANCIAL SUPPORT}

This study was supported by grants from the Heart and Stroke Foundation of Alberta, North West Territories, and Nunavut, and summer studentships funded by the Markin Undergraduate Student Research Program and O'Brien Centre to M.M. and E.F., respectively.

Disclosure: The authors declared no conflict of interest.

\section{REFERENCES}

1. Volpe JJ. Perinatal brain injury: from pathogenesis to neuroprotection. Ment Retard Dev Disabil Res Rev 2001;7:56-64.

2. Folkerth RD. The neuropathology of acquired pre- and perinatal brain injuries. Semin Diagn Pathol 2007;24:48-57.

3. Kirton A, deVeber G. Advances in perinatal ischemic stroke. Pediatr Neurol 2009;40:205-14. 
4. Bennet L, Booth L, Gunn AJ. Potential biomarkers for hypoxic-ischemic encephalopathy. Semin Fetal Neonatal Med 2010;15:253-60.

5. Perlman M, Shah PS. Hypoxic-ischemic encephalopathy: challenges in outcome and prediction. J Pediatr 2011;158:Suppl 2:e51-4.

6. Lawrence RK, Inder TE. Anatomic changes and imaging in assessing brain injury in the term infant. Clin Perinatol 2008;35:679-93, vi.

7. Counsell SJ, Tranter SL, Rutherford MA. Magnetic resonance imaging of brain injury in the high-risk term infant. Semin Perinatol 2010;34:67-78.

8. Ferriero DM, Miller SP. Imaging selective vulnerability in the developing nervous system. J Anat 2010;217:429-35.

9. Ment LR, Hirtz D, Hüppi PS. Imaging biomarkers of outcome in the developing preterm brain. Lancet Neurol 2009;8:1042-55.

10. Boardman JP, Ganesan V, Rutherford MA, Saunders DE, Mercuri E, Cowan F. Magnetic resonance image correlates of hemiparesis after neonatal and childhood middle cerebral artery stroke. Pediatrics 2005;115:321-6.

11. Lee J, Croen LA, Lindan C, et al. Predictors of outcome in perinatal arterial stroke: a population-based study. Ann Neurol 2005;58:303-8.

12. Domi T, deVeber G, Shroff M, Kouzmitcheva E, MacGregor DL, Kirton A. Corticospinal tract pre-wallerian degeneration: a novel outcome predictor for pediatric stroke on acute MRI. Stroke 2009;40:780-7.

13. Kirton A, Shroff M, Visvanathan T, deVeber G. Quantified corticospinal tract diffusion restriction predicts neonatal stroke outcome. Stroke 2007;38:974-80.

14. Lama S, Qiao M, Kirton A, et al. Imaging corticospinal degeneration in neonatal rats with unilateral cerebral infarction. Exp Neurol 2011;228:192-9.

15. Tofts PS. MT: magnetization transfer. In: Tofts PS, ed. Quantitative MRI of the Brain: Measuring Changes Caused by Disease. Chichester, UK: Wiley, 2003:257-98.

16. Henkelman RM, Stanisz GJ, Graham SJ. Magnetization transfer in MRI: a review. NMR Biomed 2001;14:57-64.

17. Jiang Q, Ewing JR, Zhang ZG, et al. Magnetization transfer MRI: application to treatment of middle cerebral artery occlusion in rat. J Magn Reson Imaging 2001;13:178-84.

18. Tuor UI, Meng S, Qiao M, et al. Differential progression of magnetization transfer imaging changes depending on severity of cerebral hypoxic-ischemic injury. J Cereb Blood Flow Metab 2008;28:1613-23.

19. Kato Y, Matsumura K, Kinosada Y, Narita Y, Kuzuhara S, Nakagawa T. Detection of pyramidal tract lesions in amyotrophic lateral sclerosis with magnetization-transfer measurements. AJNR Am J Neuroradiol 1997;18:1541-7.

20. Tanabe JL, Vermathen M, Miller R, Gelinas D, Weiner MW, Rooney WD. Reduced MTR in the corticospinal tract and normal
T2 in amyotrophic lateral sclerosis. Magn Reson Imaging 1998;16: 1163-9.

21. da Rocha AJ, Oliveira AS, Fonseca RB, Maia AC Jr, Buainain RP, Lederman HM. Detection of corticospinal tract compromise in amyotrophic lateral sclerosis with brain MR imaging: relevance of the T1-weighted spin-echo magnetization transfer contrast sequence. AJNR Am J Neuroradiol 2004;25:1509-15.

22. Lexa FJ, Grossman RI, Rosenquist AC. Dyke Award paper. MR of wallerian degeneration in the feline visual system: characterization by magnetization transfer rate with histopathologic correlation. AJNR Am J Neuroradiol 1994;15:201-12.

23. Vannucci RC, Lyons DT, Vasta F. Regional cerebral blood flow during hypoxia-ischemia in immature rats. Stroke 1988;19:245-50.

24. Vannucci RC, Vannucci SJ. Perinatal hypoxic-ischemic brain damage: evolution of an animal model. Dev Neurosci 2005;27:81-6.

25. Qiao M, Malisza KL, Del Bigio MR, Tuor UI. Correlation of cerebral hypoxic-ischemic T2 changes with tissue alterations in water content and protein extravasation. Stroke 2001;32:958-63.

26. Qiao M, Malisza KL, Del Bigio MR, Tuor UI. Transient hypoxia-ischemia in rats: changes in diffusion-sensitive MR imaging findings, extracellular space, and $\mathrm{Na}+\mathrm{K}+$-adenosine triphosphatase and cytochrome oxidase activity. Radiology 2002;223:65-75.

27. Meng S, Qiao M, Foniok T, Tuor UI. White matter damage precedes that in gray matter despite similar magnetic resonance imaging changes following cerebral hypoxia-ischemia in neonatal rats. Exp Brain Res 2005;166:56-60.

28. Kerschensteiner M, Schwab ME, Lichtman JW, Misgeld T. In vivo imaging of axonal degeneration and regeneration in the injured spinal cord. Nat Med 2005;11:572-7.

29. Nikic I, Merkler D, Sorbara C, et al. A reversible form of axon damage in experimental autoimmune encephalomyelitis and multiple sclerosis. Nat Med 2011;17:495-9.

30. da Rocha AJ, Maia AC Jr, da Silva CJ, et al. Pyramidal tract degeneration in multiple system atrophy: the relevance of magnetization transfer imaging. Mov Disord 2007;22:238-44.

31. Haynes RL, Borenstein NS, Desilva TM, et al. Axonal development in the cerebral white matter of the human fetus and infant. J Comp Neurol 2005;484:156-67.

32. Sun SW, Liang HF, Cross AH, Song SK. Evolving Wallerian degeneration after transient retinal ischemia in mice characterized by diffusion tensor imaging. Neuroimage 2008;40:1-10.

33. Paxinos G, Watson C. The Rat Brain in Sterotaxic Coordinates, 4th edn. New York: Academic Press, 1998. 